\title{
Adolescents' and parents' anxiety during COVID-19: is there a role of cyberchondriasis and emotion regulation through the internet?
}

\author{
Gülendam Akgül ${ }^{1}$ (D) - Derya Atalan Ergin $^{2}$ (D) \\ Accepted: 26 November 2020 / Published online: 3 January 2021 \\ (C) The Author(s), under exclusive licence to Springer Science+Business Media, LLC part of Springer Nature 2021
}

\begin{abstract}
COVID-19 pandemic period presents a unique context for the investigation of anxiety symptoms among adolescents and their parents. This study investigated adolescents' and their parents' anxiety symptoms, the effects of parental cyberchondriasis and adolescents' emotion regulation on anxiety symptoms. The sample consisted of 155 adolescents $(\overline{\mathrm{x}}=14.63, \mathrm{SD}=2.04)$ and one of their parents $(N=155)$. The results showed that after controlling for adolescents' gender and emotion regulation, parental cyberchondriasis and anxiety accounted for an important variance in adolescents' anxiety. Especially higher parental anxiety and compulsion were associated with higher anxiety, whereas higher distress was associated with lower anxiety. Besides, two dimensions of cyberchondriasis, compulsion, and distress, together with adolescent anxiety, predicted parental anxiety during COVID-19. While compulsion was negatively associated with anxiety, distress, and adolescent anxiety were positively associated with it. The dimensions of cyberchondriasis affected anxiety differently among adolescents and their parents. The results were discussed in terms of the implications for intervention from the ecological viewpoint.
\end{abstract}

Keywords Anxiety $\cdot$ Adolescent $\cdot$ Parents $\cdot$ Cyberchondria $\cdot$ Emotion regulation through internet

The world has faced many complex pandemics and epidemics diseases throughout history (World Health Organization [WHO], 2020a). Among these influenzas and pandemics, H1N1 caused the highest number of deaths between 150.000 and 575.000 since 2009. Whereas, the last pandemic named COVID-19 (2019-nCoV, Coronavirus) caused the death of 342.029 people. The number of cases is approximately five million since the detection of the first case in China on December 31, 2019 (WHO, 2020b). Nevertheless, there are no specific vaccines or treatments for it. WHO (2020c) suggested some precautions for people to protect themselves and for the spread of the COVID-19. Many countries have aimed to reduce the spread of the outbreak by enforcing curfews, closing workplaces, and schools. On March 16, a week after the first case detection, all schools were shut-down in Turkey.

Gülendam Akgül

gulendamakgul@karatekin.edu.tr

Derya Atalan Ergin

deryaatalan@gmail.com

Çankırı Karatekin University, Çankırı, Turkey

2 National Ministry of Education, Ankara, Turkey
A partial curfew was announced for those under 20 and over 65 years of age in some cities.

The COVID-19 had an impact on all people's psychological well-being due to the increased death rates, no available treatment options, and the changes in daily routine caused by the curfew. Anxiety, as the most common impact (WHO, 2020a), has been seen in different samples such as health professionals (Li et al., 2020; Joob \& Wiwanitkit, 2020), community sample (Ahorsu et al., 2020; Roy, 2020) and college students (Cao et al., 2020). It is an especially significant predictor of public adoption of preventive measures (Rubin et al., 2009; Gaygisiz et al., 2012).

Beyond this virus, anxiety is the most widespread mental health disorder during adolescence (Polanczyk et al., 2015; Whitney et al., 2019). Various factors have been associated with both the development and maintenance of anxiety, among which parental variables have significant importance (Hettema et al., 2001; Gamliel et al., 2018). Parents, the most important actors in children's and adolescents' microsystems according to Ecological Theory (Bronfenbrenner, 1977, 1979), have a direct influence on the psychological well being of children and adolescents and constitute as a risk factor for children's anxiety (Donovan \& Spence, 2000; Ginsburg et al., 2015; Micco et al., 2020; Rapee, 2012). Thus it is crucial to 
identify common determinants of anxiety during pandemic among adolescents and their parents.

Studies consistently supported the association between different types of anxiety in parents and offsprings (Ranøyen et al., 2015; Sackl-Pammer et al., 2015). The relationship between child and parent anxiety can be explained by genetic inheritance, environmental mechanisms in which behaviors of children and parents encourage anxiety in each other, and a shared negative environment (Ahmadzadeh et al., 2019). Therefore, it is likely that anxiety among parents and adolescents would likely affect each other during COVID-19.

Other parental psychopathologies are associated with adolescents' anxiety. Cyberchondria is a term derived from hypochondriasis and defined as the online search for health information and illnesses caused by excessive and chronic worry about being seriously ill (Starcevic \& Berle, 2013). It is a multidimensional concept. Compulsion refers to "an undesirable aspect of performing online health searches". Distress refers to "the negative emotional states and physiological reactions associated with online health searches such as sleep difficulties and anxiety". Excessiveness refers to "the time-consuming and repetitive qualities of online health searches" (Erdogan \& Hocaoglu, 2020). Two other dimensions are "seeking assurance from a medical profession" and "mistrust to medical professions (either doctors or Internet search results)" are not included in the current study since the patient density at hospitals during COVID-19 made it challenging to contact medical staff. Despite its high correlation with health anxiety, it is a distinct concept which causes significant functional impairments and increased use of health services (Mathes et al., 2018). During the COVID-19 pandemic, people are more likely to interpret body sensations as symptoms (Blakey \& Abramowitz, 2017). These interpretations may foster their emotional distress, especially health anxiety (Jungmann et al., 2020). Cyberchondriasis and health-related Internet use escalate health anxiety (Jungmann \& Witthöft, 2020; McMullan et al., 2019). Thus, this concept has special significance during the COVID-19 process.

Cyberchondriasis may affect not only themselves but also their offsprings. It was shown that the diagnosis of somatoform disorders in parents was associated with higher body preoccupations and disease phobias (Marshall et al., 2007). Thus, the online search of parents for COVID-19 related symptoms and heightened health concerns are likely to influence children's online behaviors, attitude toward pandemic and their anxiety level. Due to heightened health concerns, the pandemic period presents a unique context, especially for investigating of the effects of parental cyberchondriasis on the offsprings' anxiety. Although parental health anxiety and child anxiety were associated (Wright et al., 2017), to date, no study has investigated the effects of parental cyberchondriasis on children's anxiety level.
Increased use of the Internet has been another critical issue adolescents faced during the COVID-19 due to the increased time spent at home, social distance precautions, restrictions on the face to face communications and the limited number of indoor activities. A recent study suggested that the relation between Internet use and anxiety is minimal (Orben \& Przybylski, 2019). However, maladaptive emotion dysregulation strategies was consistently associated with increased anxiety during adolescence (Schäfer et al., 2017; Young et al., 2019). The use of the Internet and other technological tools can help get away from reality and negative emotions such as the negative affect associated with the pandemic. In other words, it serves as a kind of emotion regulation (Whang et al., 2003; Young, 1999). It is also known that the use of Internet for the emotion regulation purposes is related to wellbeing (Huang, 2010). Thus, it is expected that the use of Internet for emotion regulation purposes might be related to anxiety during the COVID-19 pandemic.

Pandemic can lead to negative emotional consequences such as depression and anxiety (Liao et al., 2014; Van Bortel et al., 2016). The rate of mental disorders increased during outbreak times (Zhou et al., 2020). Both previous studies and studies during the COVID-19 pandemic reported that media consumption such as increased use of social media and exposure to corona-related news was associated with anxiety symptoms (Gao et al., 2020a and b; MoghanibashiMansourieh, 2020; Purohit et al., 2018; Roy et al., 2020). Although there is no clear association between Internet use and anxiety, Internet is sometimes used as a tool for avoiding negative mood states, which is a kind of emotion regulation (Tokunaga, 2014). Thus, it is supposed that COVID-19 and the associated mental health problems, especially anxiety, would be related to emotion regulation through the Internet use of adolescents.

In addition to the role of the variables mentioned earlier, gender is an essential determinant of anxiety. Studies consistently reported a vulnerability for girls to anxiety symptoms (Bender et al., 2012; Lewinsohn et al., 1998). The gender differences have been supported in longitudinal studies (Duchesne \& Ratelle, 2016; Gao et al., 2020a and b). Previous studies revealed that girls had higher anxiety in terms of symptoms whereas boys had a more stable course of anxiety. (Ohannessian et al., 2017). Gender differences were also reported in many studies for the adult population (Burani, \& Nelson, 2020; MacSwain, et al., 2009). Thus they are likely to impact both adolescents' and their parents' anxiety levels during COVID-19 process.

The age is a risk factor for COVID-19 (WHO, 2020d). Older people are more likely to have a higher anxiety level than younger ones (Huang \& Zhao, 2020; Mazza et al., 2020). Adolescents are not in a high risk group and appear to experience less severe symptoms or asymptomatic cases of COVID-19 compared to adults (Banerjee et al., 2020; 
Dong et al., 2020). However, they are more likely to engage in behaviors that contribute to the spread of the infection (Oosterhoff \& Palmer, 2020). Thus, it is thought that parents but not adolescent would be affected by having an acquaintance with COVID-19.

As a result of the above studies, the present study aimed to investigate the factors related to COVID-19 anxiety among adolescents and their parents. This study answers three research questions: (1) "Do anxiety of adolescents and parents differ according to gender and COVID-19 diagnosis in acquaintances?", (2) "How much of the variance in adolescent anxiety is explained by parental cyberchondriasis, parental anxiety and emotion regulation through Internet use after controlling gender and COVID-19 diagnosed acquaintances?", (3) "How much of the variance in parental anxiety is explained by parental cyberchondriasis, adolescent anxiety, and adolescents' emotion regulation through Internet use after controlling gender and COVID-19 diagnosed acquaintances?"

As suggested in the previous literature, due to the association between parental psychopathologies and children's anxiety, (Marshall et al., 2007; Sackl-Pammer et al., 2015; Wright et al., 2017), we hypothesized that both parental cyberchondriasis and anxiety would predict adolescents' anxiety. Similarly, we expected that emotion regulation through the Internet would predict the anxiety among adolescents based on the previous associations reported between emotion regulation and well-being (Huang, 2010). Regarding the second research question, we hypothesized that cyberchondriasis would predict the anxiety among parents, as suggested earlier (Jungmann \& Witthöft, 2020; McMullan et al., 2019; Starcevic \& Berle, 2013). Moreover, adolescents' anxiety would impact parents' anxiety, although a limited number of studies have reported this association so far (eg., Ahmadzadeh et al., 2019; Sackl-Pammer et al., 2015). Adolescents' emotion regulation through the Internet was assumed to increase parents burden during the COVID-19 process and would be associated with increased anxiety of parents.

\section{Method}

\section{Procedure}

The study was conducted during the COVID-19 pandemic period. Ethical Permission was obtained from the University. Adolescents were on curfew for about five weeks when the data was collected. Three secondary schools and three high schools in Ankara's central districts were contacted and informed about the study. After the consent of school principles, teachers sent a link to the parents using class WhatsApp groups. The online link contains informed consent and survey questionnaires of a Google Forms document. The purpose of the study, the opportunity to discontinue at any time and confidentiality were explained at the beginning of the two parts of the questionnaire (for parents and adolescents separately). Parents and adolescents who responded to the survey answered the survey questions independently. Adolescents filled the second part of the questionnaire after one of their parents filled the first part.

\section{Participants}

The study group consisted of adolescents $(N=155)$ and their parents $(\mathrm{N}=155)$ who agreed to participate. The minimum sample size for the study was calculated as 106 by using the formula of " $50+8 \mathrm{~m}$ " where " $\mathrm{m}$ " is the number of factors (in the present study $m=6$ ) as suggested by Tabachnick and Fidell (2013). The voluntary parents with a child between the ages of 12 and 18 participated in the study. The parents who were diagnosed with COVID-19 were excluded from the study.

\section{Measures}

Demographic Information Parents reported on their demographic information, including their gender, education level, acquaintances with a COVID-19 diagnosis, and presence of chronic illness. Adolescents reported their age, gender, and risk perception about being infected from COVID-19. The presence of chronic illness and adolescents' risk perception did not significantly correlate with other variables and was excluded from further analysis.

Anxiety Anxiety was measured using the six-item version of the Spielberger' State-Trait Anxiety Inventory (Marteau \& Bekker, 1992). Both parents and adolescents stated how they had been feeling during the pandemic period using six items (Each item was responded to on a 4-point-Likert-type rating scale ranging from 1 (not at all) to 4 (very much). The scores range from 20 to 80 . Scores between 20 and 49 indicates the absence of anxiety, 50-59 indicates a moderate level of anxiety, and 60-80 indicates an elevated level of anxiety. Cronbach Alpha internal consistency coefficients were .86 for parents and .83 for adolescents in this study.

Emotion Regulation through Internet Usage Emotion Regulation of adolescents was measured using Emotion Regulation Subscale of Dysfunctional Internet Usage Questionnaire, which was developed by Atalan Ergin and Kapç1 (2019). It consisted of 5 items. The items were answered on a 5-point Likert-type scale, ranging from never true (1) to always true (5). Higher scores reflected greater emotion regulation through the Internet. Sample items include "I go online to relax when I feel sad, anxious or frustrated". In the current study, Cronbach Alpha's internal consistency coefficient was .81. 
Cyberchondria Parents' excessive and continuous worries about illnesses were measured by Cyberchondria Severity Scale (McElroy \& Shevlin, 2014). The scale was adapted to Turkish by Uzun and Zencir (2018). It consisted of 33 items and five subscales: compulsion, distress, excessiveness, reassurance seeking, and mistrust of medical professional. The first three subscales were used for this study. In previous studies, the subscale "mistrust" tended to be a separate construct, so that this subscale was excluded in the present study and in previous studies (Jungmann \& Witthöft, 2020). Seeking assurance subscale was not included either, as it is difficult to visit health services during the pandemic processes. The items were answered on a 3-point Likert-type scale, ranging from never (1) to always (5). Sample items for compulsion dimension "Researching symptoms or perceived medical conditions online interrupt my online leisure activities (e.g., streaming movies)", for distress "I have trouble relaxing after researching symptoms or perceived medical conditions online" and for excessiveness "If I notice an unexplained bodily sensation I will search for it on the Internet". Cronbach Alpha internal consistency coefficients were .89 for compulsion, .90 for distress, and .84 for excessiveness dimensions.

\section{Data Analysis}

Mean differences according to gender, and COVID-19 diagnosis among acquaintances were examined using independent samples $t$-tests to address the first research question. Regarding the second research question a regression model was estimated to predict adolescents' anxiety levels. The gender and presence of COVID-19 diagnosis among acquaintances were controlled in the first step, VIF was 1.04, Tolerance was .95 for both variables. Adolescent emotion regulation through the Internet was entered in the second step, VIF ranged from 1.00-1.05; Tolerance ranged from .94-.99. Parent related variables, which were compulsion, distress, excessiveness, and anxiety, were entered in the last step, VIF ranged from 1.05-2.12; Tolerance ranged from .47-.94. Before the regression analysis, preliminary analyses revealed no violations to the assumptions of homoscedasticity, normality, or linearity.

To address the third research question we estimated a regression model in which parental anxiety was predicted from the dimensions of cyberchondriasis and adolescent related variables. Similar to the first model, gender and COVID-19 diagnosis in acquaintances were controlled in the first step since the anxiety levels differed according to the gender and presence of COVID-19 diagnosis among acquaintances. In the model, control variables were entered in the first step, VIF was 1.01, Tolerance was .98 for both variables. Compulsion, distress, excessiveness were entered in the second step, VIF ranged from 1.02-1.92; Tolerance ranged from .51-.98. In the last step, adolescent anxiety and emotion regulation through the Internet were entered, VIF ranged from 1.09-1.96; Tolerance ranged from .50-.91.

\section{Results}

The adolescent group consisted of 93 females (60\%) and 62 males $(40 \%)$ whose ages ranged from 12 to $18(\mathrm{M}=14.63$, $\mathrm{SD}=2.04$ years) with a slightly positively skewed distribution.

The parents consisted of whom 129 female (83.2\%) and 26 male $(16.8 \%)$. Parents were from various educational levels (11\% primary school, $7.7 \%$ secondary school, $20.6 \%$ high school, $40 \%$ undergraduate education, $11 \%$ graduate education, and $9.7 \% \mathrm{PhD}$ education). The percentage of parents with an acquaintance with the diagnosis of COVID-19 was 23.9 .

\section{Descriptive Statistics and Preliminary Analyses}

The means, standard deviations, and bivariate correlations between the variables are presented in Table 1. As expected, mean anxiety scores in the current study were higher than the norms (approximately 36 for adults and 39-40 for adolescents, as stated by Spielberger et al., 1983), especially for parents. In order to see the mean differences according to gender, independent samples $t$-test was used. The results showed that there was a significant effect of gender on the anxiety of parents $\left(\mathrm{t}_{(153)}=-2.394, p<.05\right)$ and adolescents $\left(\mathrm{t}_{(153)}=-3.102, \mathrm{p}<.05\right)$. Women reported higher anxiety $(\overline{\mathrm{x}}=52.63)$ than men $(\overline{\mathrm{x}}=45.26)$. Similarly, girls reported higher anxiety $(\overline{\mathrm{x}}=49.68)$ than boys $(\bar{x}=41.99)$. There were no gender differences in parental compulsion, distress, excessiveness. Likewise, emotion regulation through the Internet did not differ by gender. There were no mean differences according to the presence of COVID-19 diagnosed acquaintances in adolescents' anxiety, compulsion, distress and excessiveness of parents, and adolescent emotion regulation through the Internet. However, parents who knew acquaintances with COVID-19 diagnosis had significantly higher anxiety $(\overline{\mathrm{x}}=57.61, \mathrm{SD}=12.78)$ than those who did not $(\overline{\mathrm{x}}=$ $50.42, \mathrm{SD}=14.62),\left(\mathrm{t}_{(153)}=-2.13, p<.05\right)$.

Inspection of bivariate correlations showed that parental compulsion (but not excessiveness or distress), parental anxiety and adolescent emotion regulation had significant positive correlations with adolescent anxiety symptoms. Besides, adolescent anxiety, all cyberchondria dimensions and adolescent emotion regulation through Internet had significant positive correlations with the parental anxiety symptoms. 
Table 1 Means, standard deviations and correlations between the variables of the study

\begin{tabular}{lllllllll}
\hline & 1 & 2 & 3 & 4 & 5 & 6 & Mean & SD \\
\hline 1. Parent anxiety & 1 & $.16^{*}$ & $.30^{* *}$ & $.42^{* *}$ & $.51^{* *}$ & $.25^{* *}$ & 51.39 & 14.55 \\
2. Parental compulsion & & & $.43^{* *}$ & $.60^{* *}$ & $.16^{*}$ & .06 & 12.50 & 5.55 \\
3. Parental excessiveness & & & & $.54^{* *}$ & .09 & .03 & 22.43 & 7.13 \\
4. Parental distress & & & & & .14 & .14 & 17.72 & 7.25 \\
5. Adolescent anxiety & & & & & & $.25^{* *}$ & 46.60 & 15.53 \\
6. Adolescent emotion regulation through & & & & & & 8.00 & 3.78 \\
Internet & & & & & & & \\
\hline
\end{tabular}

$* p<.05, * * p<.01, * * * p<.001$

\section{Predictors of Adolescent Anxiety}

The results of hierarchical multiple regression (Table 2) revealed that at stage one, adolescent gender contributed significantly to the regression model $F_{(2,152)}=4.93$, $p<.001$ and accounted for $6 \%$ of the variation in anxiety scores of adolescents. On the other hand, COVID-19 diagnosed acquaintances did not have a significant effect on anxiety. Introducing adolescent emotion regulation through the Internet in the second step, explained an additional $6 \%$ of the variation in anxiety and this change was significant, $F_{(3,151)}=7.25, p<.001$. Specifically, the more adolescents did emotion regulation through the Internet, the higher anxiety they had, $\beta=.25, p<.001$. Finally, the addition of parental variables to the regression model explained an additional $25 \%$ of variation in anxiety level of adolescents and this change in $\mathrm{R}^{2}$ was also significant, $F_{(7,147)}=12.82, p<.001$. Increased parental compulsion $(\beta=.22, \mathrm{p}<.001)$ and anxiety $(\beta=.55, \mathrm{p}<.001)$ was associated with increased adolescent anxiety. On the other hand, increased parental distress $(\beta=-.21, \mathrm{p}<.001)$ was associated with lower adolescent anxiety. In the last step, parental excessiveness was not a significant predictor of anxiety, $\beta=-.05, p>.05$.

Table 2 Results of hierarchical regression analyses regressing adolescents' anxiety

\begin{tabular}{lcll}
\hline Predictors & Step 1 & Step 2 & Step 3 \\
\hline Adolescent gender & $-.25^{* *}$ & $-.25^{* *}$ & $-.24^{* *}$ \\
COVID-19 diagnosed acquaintances & .04 & .02 & -.06 \\
Adolescent emotion regulation on Internet & & $.25^{* *}$ & $.13^{*}$ \\
Parental compulsion & & & $.22^{* *}$ \\
Parental distress & & & $-.21^{* *}$ \\
Parental excessiveness & & & -.05 \\
Parent anxiety & & & $.55^{* *}$ \\
$\Delta R^{2}$ & .06 & $.06^{* *}$ & $.25^{* *}$ \\
Total adjusted $\mathrm{R}^{2}$ & $.04^{* *}$ & $.10^{* *}$ & $.35^{* *}$ \\
\hline
\end{tabular}

$* p<.05, * * p<.01, * * * p<.001$

\section{Predictors of Parental Anxiety}

The third research question aimed to identify factors predicting the parental anxiety. The results of hierarchical multiple regression (Table 3) revealed that at stage one, parent gender and presence of COVID-19 diagnosed acquaintances contributed significantly to the regression model $F_{(2,152)}=6.08, p<.001$ and accounted for $7 \%$ of the variation in anxiety scores of parents. Being a female $(\beta=-.21, \mathrm{p}<.001)$ and having a COVID-19 diagnosed acquaintances $(\beta=.19, p<.001)$ were associated with higher anxiety. Introducing parental cyberchondriasis variables to the regression model explained an additional $17 \%$ of variation in anxiety level and this change in $\mathrm{R}^{2}$ was also significant, $F_{(5,149)}=10.05, p<.001$. Increased parental distress $(\beta=.40, \mathrm{p}<.001)$ was associated with higher anxiety among parents. Neither parental compulsion $(\beta=-.11$, $p>.05)$ nor parental excessiveness $(\beta=.13, p>.05)$ had significant main effects on anxiety. In the last step, introducing adolescent emotion regulation through the Internet and adolescent anxiety explained an additional $21 \%$ of the variation in anxiety and this change was significant, $F_{(7,147)}=18.14$, $p<.001$. Specifically, higher anxiety among adolescents was associated with higher anxiety among their parents $(\beta=.44, \mathrm{p}$ $<.001)$. On the other hand, adolescent emotion regulation through the Internet did not have a significant effect on parental anxiety, $\beta=.08, p>.05$.

\section{Discussion}

The role of parental anxiety and cyberchondriasis on adolescents' COVID-19 related anxiety was unknown. The role of adolescents' anxiety and emotion regulation on parental anxiety was not known, either. In the present study, we have addressed the predictive role of parent and adolescent related variables on adolescents' and parents' anxiety during the COVID-19 pandemic. Specifically, we looked at the differences according to gender and acquaintances with COVID-19 diagnosis.

Preliminary analyses revealed gender differences in anxiety scores of both adolescents and parents regarding the first 
Table 3 Results of hierarchical regression analyses regressing parents' anxiety

\begin{tabular}{lccc}
\hline Predictors & Step 1 & Step 2 & Step 3 \\
\hline Parent's gender & $-.21^{*}$ & -.14 & -.07 \\
COVID-19 diagnosed acquaintances & $.19^{*}$ & $.19^{*}$ & $.18^{*}$ \\
Parental compulsion & & -.11 & $-.18^{*}$ \\
Parental distress & & $.40^{* *}$ & $.38^{* *}$ \\
Parental excessiveness & & .13 & .13 \\
Adolescent anxiety & & & $.44^{* *}$ \\
Adolescent emotion regulation through the Internet & & .08 \\
$\Delta R^{2}$ & $.07^{* *}$ & $.17^{* *}$ & $.21^{* *}$ \\
Total adjusted $\mathrm{R}^{2}$ & $.06^{* *}$ & $.22^{* *}$ & $.43^{* *}$ \\
\hline
\end{tabular}

$* p<.05, * * p<.01, * * * p<.001$ research question. The results were consistent with previous findings, which presented higher anxiety scores for females in adolescence (Bender et al., 2012; Lewinsohn et al., 1998) and in the adult population (Burani \& Nelson, 2020; MacSwain et al., 2009). Although the differences were said to be due to a heightened sensitivity of females to the threat (Burani \& Nelson, 2020), the result has implications for the identification of risk groups and interventions.

Likewise, the presence of COVID-19 diagnosed acquaintances predicted anxiety among parents but not among adolescents. As suggested earlier, age matters in terms of COVID-19 anxiety (Cao et al., 2020; Mazza et al., 2020). Similarly, direct experience with the Swine Flu did not influence children's fears (Remmerswaal \& Murris, 2011). The result demonstrated that adolescents did not perceive a threat for COVID-19, but their parents did. Thus, adolescents should be targeted for awareness-raising against the COVID-19 since they are likely to play a role in spreading it (Oosterhoff et al., 2020).

Regarding the second research question, in line with the previous findings, parental anxiety predicted adolescents' anxiety (Ahmadzadeh, 2019; Ranøyen et al., 2015; SacklPammer et al., 2015). Secondly, emotion regulation through the Internet positively predicted adolescents' anxiety. The previous studies on the relationship between Internet usage and anxiety presented contradictory findings. Some studies supported this relationship (Akin \& Iskender, 2011; Kim \& Davis, 2009; Schäfer et al., 2017; Young et al., 2019), whereas other studies showed that the relationship is too small to consider (Orben \& Przybylski, 2019). This finding is in line with former studies. Emotion regulation through the Internet was a subscale of problematic Internet usage, which is also related to decreased well-being (Caplan, 2002) and anxiety (Mojtabai et al., 2016; Spada et al., 2008). The preference for online social interaction predicted deficiency in self-regulation and emotion regulation on the Internet (Caplan, 2010). Adolescents tended to use the Internet for emotion regulation purposes and social interaction more than other ages (Lehman, 2012). Thus, the findings supported that adolescents who tried to regulate their feelings through the Internet had higher anxiety during the pandemic. Additionally, because of the correlational nature of the findings, it is also possible that anxious adolescents might use the Internet to regulate their anxiety.

When we looked at the effect of parental cyberchondriasis on adolescent anxiety, our findings suggested that after controlling for adolescents' gender and emotion regulation, parental anxiety and cyberchondriasis accounted for an important variance on adolescents' anxiety. Especially higher parental anxiety and compulsion were associated with higher adolescent anxiety, whereas higher distress was associated with lower adolescent anxiety. Excessiveness was not associated with adolescent anxiety. Parental compulsion may be likely to interrupt activities of daily living due to constant web search. Another explanation might be that parents with higher compulsion may focus on their own emotions and spend more time on the Internet to search for COVID-19. Thus, they may ignore their children. Compulsion is also said to increase worry and anxiety due to the huge number of information they faced with the Internet during COVID-19 (Kavi \& Bazrafshan, 2020). Although the association between parental cyberchondriasis and anxiety among their children was not investigated priorly, the current results suggested compulsively search for health information by parents was likely to increase anxiety among adolescents. On the other hand, parental distress was associated with lower anxiety among adolescents. It seems that distress experienced by parents provides an opportunity in the family to share, discuss, and normalize the COVID-19 process and associated negative feelings (WHO, 2020a).

Regarding the third research question, we examined the predicting role of adolescent related variables and parental cyberchondriasis on parents' anxiety levels. First of all, adolescents' anxiety significantly predicted their parents' anxiety. Although not many previous studies investigated this relationship, child anxiety symptoms were shown to predict higher anxiety among mothers (Ahmadzadeh et al., 2019; SacklPammer et al., 2015). This finding deserves further attention despite the cross-sectional design did not allow for a causal 
inference. Secondly, adolescent emotion regulation did not predict the parents' anxiety on the contrary to expectations. It might be possible that many adolescents may have used the Internet to regulate emotions due to curfews and the limited number of activities available at home.

Finally, the effects of cyberchondriasis on parental anxiety were as expected. Two dimensions of cyberchondriasis, compulsion and distress, predicted parental anxiety during COVID-19. While compulsion was negatively associated with anxiety, distress was positively associated with parental anxiety. The finding that dimensions of cyberchondria predicted anxiety is similar to findings from Jungmann and Witthöft (2020), who also reported significant correlations between anxiety during the pandemic and the dimensions of cyberchondria. However, the results differ from theirs in that compulsive search on Internet decreased parents' anxiety level in the present study. The present study's different findings might be because the perceived sufficiency of information related to pandemic reduces the worry (Goulia et al., 2010). A study during a previous pandemic reported that exposure to television was associated with higher anxiety (Van den Bulck $\&$ Custers, 2009). Thus, the content and credibility of search results or exposed materials may influence their anxiety.

Overall, when the results are evaluated together for adolescents and parents, a striking finding emerged. It is clearly seen that parental compulsion predicts higher anxiety among adolescents but lower anxiety for parents. On the contrary, parental distress predicted higher anxiety for themselves but not for adolescents. A possible reason for why compulsion and distress dimensions had different associations with adolescents' anxiety could be that compulsion is an act whereas distress is an emotion. Parents would likely consciously hide their distress from their children but this would not be the case when they consistently search for information on the media tools. Since cyberchondria is a relatively new term in the literature (Brown et al., 2019; Starcevic \& Berle, 2013), more studies are needed to reveal its effects on not only on people who suffer from it but also people around them such as their children.

In both parental anxiety and adolescent anxiety models, excessiveness was not associated with anxiety which is surprising since there were positive correlations between three dimensions of cyberchondriasis. Similarly, the similar positive correlations were reported in another study (Jungmann \& Witthöft, 2020). In both parental anxiety and adolescent anxiety models, excessiveness was not associated with anxiety. The finding is surprising since there were positive correlations between the three dimensions of cyberchondriasis. Similarly, the similar positive correlations were reported in another study (Jungmann \& Witthöft, 2020). However, in that study, the mean excessiveness scores were lower compared to the times without pandemic. The difference was attributed to the fact that possible symptoms of infection are searched online less often compared to other topics of the pandemic, and that during the COVID-19 pandemic doctors are visited less often. Besides, during the time of ambiguity, people may increase the use of media to get rid of uncertainty and to evaluate risks (Ball-Rokeach \& DeFleur, 1976; Lachlan et al., 2016). During the crisis, functional use of media can be a suitable way to get the right information (Lachlan et al., 2016). For example, high media exposure was associated with higher anxiety during the Ebola virus pandemic (Thompson et al., 2017). Thus, the use of Internet to search for the ambiguous symptoms COVID-19 may not be related to anxiety.

Implications of our study may help identify risk factors for anxiety of parents and adolescents and preventive measures during such pandemics. From an ecological viewpoint, it has been known that parents are the primary agents who shape the children (Bronfenbrenner, 1977, 1979). During the pandemic, since all the world has limited the physical connections, families have started to spend more time together. As a result, the parental influences become more and more critical for children. Therefore, parental pathologies, like cyberchondria present a significant risk factor for children. Preventive efforts or interventions should inform parents about the results of cyberchondria behaviors, especially compulsive search for online information about COVID-19. Also, they need to know that the online sources are not reliable information sources for illnesses.

Despite its important contribution to the literature, the study had some limitations. First, we used online data collection. During the COVID-19, it was almost impossible to apply paper-pencil tests due the precautions and curfew. The sample may be composed of those who were sensitive to anxiety. Also, response bias may exist due to self-report data. Adolescents and parents may have a tendency to present themselves in a more positive way and may underreport the negative emotions since they thought that their responses could be seen by each other. Finally, the present study did not focused on adolescents' own cyberchondriasis. Future studies can investigate the effects of both parental and adolescents' cyberchondriasis on anxiety using other modern analyses like actor-partner interdependence models.

Data Avalibility The data that support the findings of this study are available from the corresponding author, upon reasonable request.

\section{Compliance with Ethical Standards}

Conflict of Interest The authors declare that there is no conflict of interest.

Ethical Statement All procedures performed in studies involving human participants were in accordance with the ethical standards of the Final International University Ethics Committee.

Informed Consent Form Informed consent form was signed by the participants who voluntarily agreed to take part in this study. 


\section{References}

Ahmadzadeh, Y. I., Eley, T. C., Leve, L. D., Shaw, D. S., Natsuaki, M. N., Reiss, D., Neiderhiser, J.M. \& McAdams, T. A. (2019). Anxiety in the family: A genetically informed analysis of transactional associations between mother, father and child anxiety symptoms. Journal of Child Psychology and Psychiatry, 60(12), 1269-1277. https://doi.org/10.1111/jcpp.13068.

Ahorsu, D. K., Lin, C. Y., Imani, V., Saffari, M., Griffiths, M. D., \& Pakpour, A. H. (2020). The fear of COVID-19 scale: Development and initial validation. International Journal of Mental Health and Addiction, 1-9. https://doi.org/10.1007/s11469020-00270-8.

Akin, A., \& Iskender, M. (2011). Internet addiction and depression, anxiety and stress. International Online Journal of Educational Sciences, 3(1), 138-148.

Atalan Ergin, D., \& Kapçı, E. G. (2019). Validity and reliability study of parental mediation strategies for internet usage scale-adolescent and parent forms in the Turkish sample. Journal of Measurement and Evaluation in Education and Psychology, 10(2), 117-132. https:// doi.org/10.21031/epod.457218.

Banerjee, A., Pasea, L., Harris, S., Gonzalez-Izquierdo, A., Torralbo, A., Shallcross, L., Noursadeghi, M., Pillay, D., Sebire, N., Holmes, C., Pagel, C., Wong, W. K., Langenberg, C., Williams, B., Denaxas, S., \& Hamingway, H. (2020). Estimating excess 1-year mortality associated with the COVID-19 pandemic according to underlying conditions and age: A population-based cohort study. The Lancet, 395(10238), 1715-1725. https://doi.org/10.1016/S0140-6736(20) 30854-0.

Ball-Rokeach, S. J., \& DeFleur, M. L. (1976). A dependency model of mass-media effects. Communication Research, 3(1), 3-21. https:// doi.org/10.1177/009365027600300101.

Bender, P. K., Reinholdt-Dunne, M. L., Esbjørn, B. H., \& Pons, F. (2012). Emotion dysregulation and anxiety in children and adolescents: Gender differences. Personality and Individual Differences, 53(3), 284-288. https://doi.org/10.1016/j.paid.2012.03.027.

Blakey, S. M., \& Abramowitz, J. S. (2017). Psychological predictors of health anxiety in response to the Zika virus. Journal of Clinical Psychology in Medical Settings, 24, 270-278. https://doi.org/10. 1007/s10880-017-9514-y.

Bronfenbrenner, U. (1977). Toward an experimental ecology of human development. American Psychologist, 32, 513-531. https://doi.org/ 10.1037/0003-066X.32.7.513.

Bronfenbrenner, U. (1979). Contexts of child rearing: Problems and prospects. American Psychologist, 34, 844-850. https://doi.org/10.1037/ 0003-066X.34.10.844.

Burani, K., \& Nelson, B. D. (2020). Gender differences in anxiety: The mediating role of sensitivity to unpredictable threat. International Journal of Psychophysiology, 153, 127-134. https://doi.org/10. 1016/j.ijpsycho.2020.05.001.

Cao, W., Fang, Z., Hou, G., Han, M., Xu, X., Dong, J., \& Zheng, J. (2020). The psychological impact of the COVID-19 epidemic on college students in China. Psychiatry Research, 287, 112934. https://doi.org/10.1016/j.psychres.2020.112934.

Caplan, S. E. (2002). Problematic internet use and psychosocial wellbeing: Development of a theory-based cognitive-behavioral measurement instrument. Computers in Human Behavior, 18(5), 553575. https://doi.org/10.1016/S0747-5632(02)00004-3.

Caplan, S. E. (2010). Theory and measurement of generalized problematic internet use: A two-step approach. Computers in Human Behavior, 26(5), 1089-1097. https://doi.org/10.1016/j.chb.2010. 03.012 .

Dong, Y., Mo, X., Hu, Y., Qi, X., Jiang, F., Jiang, Z., \& Tong, S. (2020). Epidemiological characteristics of 2143 pediatric patients with 2019 coronavirus disease in China. Pediatrics., 145, e20200702. https:// doi.org/10.1542/peds.2020-0702.

Donovan, C. L., \& Spence, S. H. (2000). Prevention of childhood anxiety disorders. Clinical Psychology Review, 20(4), 509-531. https://doi. org/10.1016/S0272-7358(99)00040-9.

Duchesne, S., \& Ratelle, C. F. (2016). Patterns of anxiety symptoms during adolescence: Gender differences and sociomotivational factors. Journal of Applied Developmental Psychology, 46, 41-50. https://doi.org/10.1016/j.appdev.2016.07.001.

Erdogan, A., \& Hocaoglu, C.. (2020). Cyberchondria: A Review. Current Approaches to Psychiatry, 12(4), 435-444. https://doi.org/10. 18863/pgy.654648.

Gamliel, K. H., Dollberg, D. G., \& Levy, S. (2018). Relations between parents' anxiety symptoms, marital quality, and preschoolers' externalizing and internalizing behaviors. Journal of Child and Family Studies, 27(12), 3952-3963. https://doi.org/10.1007/s10826-0181212-3.

Gao, W., Ping, S., \& Liu, X. (2020a). Gender differences in depression, anxiety, and stress among college students: A longitudinal study from China. Journal of Affective Disorders, 263, 292-300. https:// doi.org/10.1016/j.jad.2019.11.121.

Gao, J., Zheng, P., Jia, Y., Chen, H., Mao, Y., Chen, S., Mao, Y., Chen, S., Wang, Y., Fu, H., \& Dai, J. (2020b). Mental health problems and social media exposure during COVID-19 outbreak. PLoS One, 15(4), e 0231924. https://doi.org/10.1371/journal.pone.0231924.

Gaygısız, Ü., Gaygısız, E., Özkan, T., \& Lajunen, T. (2012). Individual differences in behavioral reactions to H1N1 during a later stage of the epidemic. Journal of Infection and Public Health, 5(1), 9-21.

Ginsburg, G. S., Drake, K. L., Tein, J. Y., Teetsel, R., \& Riddle, M. A. (2015). Preventing onset of anxiety disorders in offspring of anxious parents: A randomized controlled trial of a family-based intervention. American Journal of Psychiatry, 172(12), 1207-1214. https:// doi.org/10.1016/j.jiph.2011.09.008.

Goulia, P., Mantas, C., Dimitroula, D., Mantis, D., \& Hyphantis, T. (2010). General hospital staff worries, perceived sufficiency of information and associated psychological distress during the a/H1N1 influenza pandemic. BMC Infectious Diseases, 10(1), 322-332. https://doi.org/10.1186/1471-2334-10-322.

Hettema, J. M., Neale, M. C., \& Kendler, K. S. (2001). A review and meta-analysis of the genetic epidemiology of anxiety disorders. American Journal of Psychiatry, 158(10), 1568-1578. https://doi. org/10.1176/appi.ajp.158.10.1568.

Huang, C. (2010). Internet use and psychological well-being: A metaanalysis. Cyberpsychology, Behavior and Social Networking, 13(3), 241-249. https://doi.org/10.1089/cyber.2009.0217.

Huang, Y., \& Zhao, N. (2020). Mental health burden for the public affected by the COVID-19 outbreak in China: Who will be the high-risk group? Psychology, Health \& Medicine, 1-12. https:// doi.org/10.1080/13548506.2020.1754438.

Joob, B., \& Wiwanitkit, V. (2020). Traumatization in medical staff helping with COVID-19 control. Brain, Behavior, and Immunity, 87(10), 10. https://doi.org/10.1016/j.bbi.2020.03.020.

Jungmann, S. M., \& Witthöft, M. (2020). Health anxiety, cyberchondria, and coping in the current COVID-19 pandemic: Which factors are related to coronavirus anxiety. Journal of Anxiety Disorders, 73, 102239. https://doi.org/10.1016/j.janxdis.2020.102239.

Jungmann, S. M., Brand, S., Kolb, J., \& Witthöft, M. (2020). Do Dr. Google and health apps have (comparable) side effects? An experimental study. Clinical Psychological Science, 8(2), 306-317. https://doi.org/10.1177/2167702619894904.

Kavi, E., \& Bazrafshan, M. R. (2020). Cyberchondria and COVID-19 pandemic. Journal of Health Sciences \& Surveillance System, 8(2), 98-98. https://doi.org/10.30476/JHSSS.2020.86231.1085.

Kim, H. K., \& Davis, K. E. (2009). Toward a comprehensive theory of problematic internet use: Evaluating the role of self-esteem, anxiety, flow, and the self-rated importance of internet activities. Computers 
in Human Behavior, 25(2), 490-500. https://doi.org/10.1016/j.chb. 2008.11.001.

Lachlan, K. A., Spence, P. R., Lin, X., Najarian, K., \& Del Greco, M. (2016). Social media and crisis management: CERC, search strategies, and twitter content. Computers in Human Behavior, 54, 647 652. https://doi.org/10.1016/j.chb.2015.05.027.

Lehman, J. (2012). Time Inc. study reveals that "digital natives" switch between devices and platforms every two minutes, use media to regulate their mood. http://www.businesswire.com/news/home/ 20120409005536/en/Time-Study-Reveals.

Lewinsohn, P. M., Gotlib, I. H., Lewinsohn, M., Seeley, J. R., \& Allen, N. B. (1998). Gender differences in anxiety disorders and anxiety symptoms in adolescents. Journal of Abnormal Psychology, 107(1), 109-117. https://doi.org/10.1037/0021-843X.107.1.109.

Li, Z., Ge, J., Yang, M., Feng, J., Qiao, M., Jiang, R., et al. (2020). Vicarious traumatization in the general public, members, and nonmembers of medical teams aiding in COVID-19 control. Brain, Behavior, and Immunity, 88, 916-919. https://doi.org/10.1016/j. bbi.2020.03.007.

Liao, Q., Cowling, B. J., Lam, W. W., Ng, D. M., \& Fielding, R. (2014). Anxiety, worry and cognitive risk estimate in relation to protective behaviors during the 2009 influenza a/H1N1 pandemic in Hong Kong: Ten cross-sectional surveys. BMC Infectious Diseases, 14, 169-179. https://doi.org/10.1186/1471-2334-14-16926.

MacSwain, K. L. H., Sherry, S. B., Stewart, S. H., Watt, M. C., Hadjistavropoulos, H. D., \& Graham, A. R. (2009). Gender differences in health anxiety: An investigation of the interpersonal model of health anxiety. Personality and Individual Differences, 47(8), 938-943. https://doi.org/10.1016/j.paid.2009.07.020.

Marshall, T., Jones, D. P., Ramchandani, P. G., Stein, A., \& Bass, C. (2007). Intergenerational transmission of health beliefs in somatoform disorders: Exploratory study. The British Journal of Psychiatry, 191(5), 449-450. https://doi.org/10.1192/bjp.bp.107. 035261.

Marteau, T. M., \& Bekker, H. (1992). The development of a six-item short-form of the state scale of the Spielberger state - Trait anxiety inventory (STAI). British Journal of Clinical Psychology, 31(3), 301-306. https://doi.org/10.1111/j.2044-8260.1992.tb00997.x.

Mathes, B. M., Norr, A. M., Allan, N. P., Albanese, B. J., \& Schmidt, N. B. (2018). Cyberchondria: Overlap with health anxiety and unique relations with impairment, quality of life, and service utilization. Psychiatry Research, 261, 204-211. https://doi.org/10.1016/j. psychres.2018.01.002.

Mazza, C., Ricci, E., Biondi, S., Colasanti, M., Ferracuti, S., Napoli, C., \& Roma, P. (2020). A nationwide survey of psychological distress among italian people during the COVID-19 pandemic: Immediate psychological responses and associated factors. International Journal of Environmental Research and Public Health, 17(9), 3165. https://doi.org/10.3390/ijerph17093165.

McElroy, E., \& Shevlin, M. (2014). The development and initial validation of the cyberchondria severity scale (CSS). Journal of Anxiety Disorders, 28(2), 259-265. https://doi.org/10.1016/j.janxdis.2013. 12.007

McMullan, R. D., Berle, D., Arnáez, S., \& Starcevic, V. (2019). The relationships between health anxiety, online health information seeking, and cyberchondria: Systematic review and meta-analysis. Journal of Affective Disorders, 245, 270-278. https://doi.org/10. 1016/j.jad.2018.11.037.

Micco, J. A., Henin, A., Mick, E., Kim, S., Hopkins, C. A., Biederman, J., \& Hirshfeld-Moghanibashi-Mansourieh, A. (2020). Assessing the anxiety level of Iranian general population during COVID-19 outbreak. Asian Journal of Psychiatry, 102076, 102076. https://doi.org/ 10.1016/j.ajp.2020.102076.

Mojtabai, R., Olfson, M., \& Han, B. (2016). National trends in the prevalence and treatment of depression in adolescents and young adults.
Pediatrics, 138, e20161878. https://doi.org/10.1542/peds.20161878.

Ohannessian, C. M., Milan, S., \& Vannucci, A. (2017). Gender differences in anxiety trajectories from middle to late adolescence. Journal of Youth and Adolescence, 46(4), 826-839. https://doi.org/ 10.1007/s10964-016-0619-7.

Oosterhoff, B., \& Palmer, C. A. (2020). Psychological correlates of news monitoring, social distancing, disinfecting, and hoarding behaviors among US adolescents during the COVID-19 pandemic. Adolescent COVID-19 Behavior, 1-20. https://doi.org/10.31234/osf.io/rpcy4.

Oosterhoff, B., Palmer, C. A., Wilson, J., \& Shook, N. (2020). Adolescents' motivations to engage in social distancing during the COVID-19 pandemic: Associations with mental and social health. Journal of Adolescent Health, 67(2), 179-185. https://doi.org/10. 1016/j.jadohealth.2020.05.004.

Orben, A., \& Przybylski, A. K. (2019). The association between adolescent well-being and digital technology use. Nature Human Behaviour, 3(2), 173-182. https://doi.org/10.1038/s41562-0180506-1.

Polanczyk, G. V., Salum, G. A., Sugaya, L. S., Caye, A., \& Rohde, L. A. (2015). Annual research review: A meta-analysis of the worldwide prevalence of mental disorders in children and adolescents. Journal of Child Psychology and Psychiatry, 56(3), 345-365. https://doi. org/10.1111/jcpp.12381.

Purohit, V., Kudale, A., Sundaram, N., Joseph, S., Schaetti, C., \& Weiss, M. G. (2018). Public health policy and experience of the 2009 H1N1 influenza pandemic in Pune, India. International Journal of Health Policy and Management, 7(2), 154-166. https://doi.org/10.15171/ ijhpm.2017.54.

Ranøyen, I., Stenseng, F., Klöckner, C. A., Wallander, J., \& Jozefiak, T. (2015). Familial aggregation of anxiety and depression in the community: The role of adolescents' self-esteem and physical activity level (the HUNT study). BMC Public Health, 15(1), 1-16. https:// doi.org/10.1186/s12889-015-1431-0.

Rapee, R. M. (2012). Family factors in the development and management of anxiety disorders. Clinical Child and Family Psychology Review, 15(1), 69-80. https://doi.org/10.1007/s10567-011-0106-3.

Remmerswaal, D., \& Muris, P. (2011). Children's fear reactions to the 2009 swine flu pandemic: The role of threat information as provided by parents. Journal of Anxiety Disorders, 25(3), 444-449. https:// doi.org/10.1016/j.janxdis.2010.11.008.

Roy, D., Tripathy, S., Kar, S. K., Sharma, N., Verma, S. K., \& Kaushal, V. (2020). Study of knowledge, attitude, anxiety \& perceived mental healthcare need in Indian population during COVID-19 pandemic. Asian Journal of Psychiatry, 102083. https://doi.org/10.1016/j.ajp. 2020.102083.

Rubin, G. J., Amlot, R., Page, L., \& Wessely, S. (2009). Public perceptions, anxiety, and behaviour change in relation to the swine flu outbreak: Cross sectional telephone survey. BMJ, 339, 444-449. https://oi.org/10.1136/bmj.b2651.

Sackl-Pammer, P., Popow, C., Schuch, B., Aigner, M., \& Friedrich, M. \&. Huemer, J. (2015). Psychopathology among parents of children and adolescents with separation anxiety disorder. Neuropsychiatrie, 29, 23-28. https://doi.org/10.1007/s40211-014-0133-7.

Schäfer, J. Ö., Naumann, E., Holmes, E. A., Tuschen-Caffier, B., \& Samson, A. C. (2017). Emotion regulation strategies in depressive and anxiety symptoms in youth: A meta-analytic review. Journal of Youth and Adolescence, 46(2), 261-276. https://doi.org/10.1007/ s10964-016-0585-0.

Spada, M. M., Langston, B., Nikčević, A. V., \& Moneta, G. B. (2008). The role of metacognitions in problematic internet use. Computers in Human Behavior, 24(5), 2325-2335. https://doi.org/10.1016/j. chb.2007.12.002.

Starcevic, V., \& Berle, D. (2013). Cyberchondria: Towards a better understanding of excessive health-related internet use. Expert Review 
of Neurotherapeutics, 13(2), 205-213. https://doi.org/10.1586/ ERN.12.162.

Tabachnick, B. G., \& Fidell, L. S. (2013). Using multivariate statistics: International edition. Pearson2012.

Thompson, R. R., Garfin, D. R., Holman, E. A., \& Silver, R. C. (2017). Distress, worry, and functioning following a global health crisis: A national study of Americans' responses to Ebola. Clinical Psychological Science, 5, 513-521. https://doi.org/10.1177/ 2167702617692030.

Tokunaga, R. S. (2014). A unique problem or the manifestation of a preexisting disorder? The mediating role of problematic internet use in the relationships between psychosocial problems and functional impairment. Communication Research, 41(4), 531-560. https://doi.org/10.1177/0093650212450910.

Uzun, S. U., \& Zencir, M. (2018). Reliability and validity study of the Turkish version of cyberchondria severity scale. Current Psychology, 1-7. https://doi.org/10.1007/s12144-018-0001-x.

Van Bortel, T., Basnayake, A., Wurie, F., Jambai, M., Koroma, A. S., Muana, A. T., Hann, K., Eaton, J., Martin, S., \& Nellums, L. B. (2016). Psychosocial effects of an Ebola outbreak at individual, community and international levels. Bulletin of the World Health Organization, 94(3), 210-214. https://doi.org/10.2471/BLT.15. 158543.

Van den Bulck, J., \& Custers, K. (2009). Television exposure is related to fear of avian flu, an ecological study across 23 member states of the European Union. European Journal of Public Health, 19, 370-374. https://doi.org/10.1093/eurpub/ckp061.

Whang, L. S. M., Lee, S., \& Chang, G. (2003). Internet over-users' psychological profiles: A behavior sampling analysis on internet addiction. Cyberpsychology \& Behavior, 6(2), 143-150. https://doi.org/ 10.1089/109493103321640338.

Whitney, D. G., Shapiro, D. N., Warschausky, S. A., Hurvitz, E. A., \& Peterson, M. D. (2019). The contribution of neurologic disorders to the national prevalence of depression and anxiety problems among children and adolescents. Annals of Epidemiology, 29, 81-84. https://doi.org/10.1016/j.annepidem.2018.11.003.
WHO, (2020a). Mental health and psychosocial considerations during the COVID-19 outbreak.https://www.who.int/docs/default-source/ coronaviruse/mental-health-considerations.pdf.

WHO (2020b). Coronovirus disease (COVID-19) Situation Report. https://www.who.int/docs/default-source/coronaviruse/situationreports/20200525-covid-19-sitrep-126.pdf?sfvrsn=887dbd66_2\% 20community\%20and\%20international\%20levels

WHO (2020c). Coronavirus disease (COVID-19) advice for the public https://www.who.int/emergencies/diseases/novel-coronavirus2019/advice-for-public

WHO (2020d). COVID-19: vulnerable and high risk groups https:// www.who.int/westernpacific/emergencies/covid-19/information/ high-risk-groups

Wright, K. D., Reiser, S. J., \& Delparte, C. A. (2017). The relationship between childhood health anxiety, parent health anxiety, and associated constructs. Journal of Health Psychology, 22(5), 617-626. https://doi.org/10.1177/1359105315610669.

Young, K. S. (1999). Internet addiction: Symptoms, evaluation and treatment. Innovations in clinical practice: A source book, 17(17), 351352. https://doi.org/10.3390/brainsci9040076.

Young, K. S., Sandman, C. F., \& Craske, M. G. (2019). Positive and negative emotion regulation in adolescence: Links to anxiety and depression. Brain Sciences, 9(4), 76-95. https://doi.org/10.3390/ brainsci9040076.

Zhou, S. J., Zhang, L. G., Wang, L. L., Guo, Z. C., Wang, J. Q., Chen, J. C., Liu, M., Chen, X. \& Chen, J. X. (2020). Prevalence and sociodemographic correlates of psychological health problems in Chinese adolescents during the outbreak of COVID-19. European Child \& Adolescent Psychiatry, 29, 1-10. https://doi.org/10.1007/s00787020-01541-4.

Publisher's Note Springer Nature remains neutral with regard to jurisdictional claims in published maps and institutional affiliations. 